\title{
Diskussion der Ergebnisse
}

In diesem Kapitel wird diskutiert, welchen Beitrag die vorliegende Arbeit zur aktuellen Forschung leistet (vgl. Abschn. 8.1) und welche Grenzen ihr auferlegt sind (vgl. Abschn. 8.3). Darüber hinaus werden in einem weiteren Unterkapitel alle relevanten Konsequenzen und Empfehlungen für die Unterrichtspraxis erörtert (vgl. Abschn. 8.2). Zuletzt zeigt ein Ausblick Potenziale für Anschlussforschung, weitere Fragestellungen und Desiderata auf (vgl. Abschn. 8.4).

\subsection{Eigener Beitrag zum Forschungsstand}

Die vorliegende Arbeit ergänzt die aktuelle Forschungslandschaft zu sprachsensiblem Geographieunterricht in verschiedenen Aspekten. Im Folgenden wird der Mehrwert dieser Arbeit vor allem für die geographiedidaktische Forschung dargestellt. Dabei sei übergeordnet zunächst auf den methodischen Rahmen verwiesen, der die Arbeit strukturiert.

\section{Design-Based Research in der Geographiedidaktik}

Design-Based Research wird in der Geographiedidaktik bisher wenig genutzt, obschon es sich zunehmender Beliebtheit erfreut. DBR ermöglichte es, auf verschiedenen Ebenen, nämlich einerseits theoretisch für die Grundlagenforschung und andererseits für die unterrichtliche Praxis, anschlussfähige Ergebnisse zu generieren. Die zentralen Erkenntnisse, die sich aus dem methodischen Rahmen ergeben, diskutiere ich im Folgenden.

Durch den iterativen Ablauf unterscheidet sich Design-Based Research wesentlich von anderen Forschungsdesigns. Im zyklischen Vorgehen werden Daten erhoben und ausgewertet, sodass die Intervention auf dieser Basis entsprechend 
angepasst werden kann. Die Daten werden eher bottom-up, also mehrfach und formativ im Forschungsprozess erhoben. Dieses Vorgehen unterscheidet sich zentral vom dem, was üblicherweise in der Geographiedidaktik, aber auch in bildungswissenschaftlichen Studien wie PISA, TIMSS und IGLU angewendet wird. Der Prozess im Design-Based Research führt dazu, dass die Zusammenarbeit mit Praktiker*innen, in diesem Fall Geographielehrkräften, gestärkt wird und der reale Rahmen der Schule Berücksichtigung findet. Dies wiederum hat zur Folge, dass die Maßnahmen zu höherer Akzeptanz auf Seiten der Lehrkräfte führen und eine Implementierung in der Schulpraxis eher wahrscheinlich ist. So wurde die beforschte Unterrichtsreihe nach dem ersten Design-Zyklus auf Basis der Rückmeldung der Lehrkräfte sowie der Datenauswertung angepasst. Auch die Schüler*innen wurden in den Überarbeitungsprozess durch Feedbackmöglichkeiten einbezogen. Eine weitere Dimension der zyklischen, formativen Entwicklungs- und Forschungsarbeit ist die Annäherung an eine möglichst optimale Lösung. In diesem Sinne sind die Design-Kriterien und die daraus entstehende Unterrichtsreihe als Teil der Problemlösung zu begreifen - das Praxisproblem, Geographie sprachsensibel zu unterrichten. Um ein Problem zu lösen, wird auf Vorstellung(en) von einem Zielzustand zurückgegriffen, der die Problemlösehandlung maßgeblich beeinflusst. In der praktischen Umsetzung der Zielvorstellung ist es dann aber so, dass die Erwartungen nicht so getroffen werden. Die Problemlösung ist also durch unsere Vorstellung geprägt und gleichzeitig begrenzt. Simon (1955) beschreibt dieses Phänomen unter dem Begriff bounded rationality wie folgt:

Broadly stated, the task is to replace the global rationality of economic man with the kind of rational behavior that is compatible with the access to information and the computational capacities that are actually possessed by organisms, including man, in the kinds of environments in which such organisms exist. (Simon 1955, S. 99)

Diese bounded rationality lässt sich am besten durch iteratives Vorgehen in den Designprozess einbinden (also abmildern). Jeder Design-Zyklus erzeugt dabei zwar keine perfekte, jedoch eine hinreichend zufriedenstellende, also optimale Problemlösung. So wurden z. B. durch die Durchführung des ersten Design-Zyklus die Testverfahren verbessert und in Design-Zyklus II das Problem der Evidenzbeurteilung für die Unterrichtsreihe und die Reliabilitäten adressiert. Je mehr Design-Zyklen durchgeführt werden können, desto stärker kann das Phänomen der bounded rationality abgefedert werden. Die zeitlichen Ressourcen hat dieses Projekt auf zwei Design-Zyklen beschränkt.

Mit diesen fruchtbaren Ansätzen, die sich aus der Designforschung ergeben, sind jedoch auch zahlreiche Limitationen verbunden, auf die teilweise schon im 
entsprechenden Methodikkapitel eingegangen wurde (vgl. Abschn. 6.1.3). Besonders kritisch hervorzuheben ist der Bruch mit klassischen Gütekriterien. Dadurch, dass die Intervention während des Forschungsprozesses verbessert wird und die Einheit als Gesamtes betrachtet und beforscht wird, also keine isolierten Variablen im Fokus stehen, entstehen Einbußen hinsichtlich der Validität. Das Verhältnis des flexiblen, praxisnahen Forschungsdesigns DBR zu klassischen Gütekriterien wird auch unter den Grenzen des Projekts nochmals kritisch diskutiert (vgl. Abschn. 8.3).

\section{Begriffliche Klarheit}

In der Diskussion um Bildungs-, Fach- und Alltagssprache sowie angrenzende Begriffe gibt es verschiedene, inkonsistent nebeneinander existierende Definitionen und Abgrenzungsversuche. Die begriffliche Heterogenität ist im Grundlagenteil dieser Arbeit dargelegt (vgl. Abschn. 2.1). Außerdem stellt die Zusammenschau des Diskurses den Versuch dar, begriffliche Klarheit zu schaffen. Was unter Fachsprache zu fassen ist, wurde zunächst theoretisch dargelegt und über die Entwicklung bzw. Adaption der Erhebungsinstrumente im empirischen Teil operationalisiert. Die Begriffsabgrenzung sowie die Operationalisierung in dieser Arbeit stellen einen von mehreren Zugängen dar. Ebenso gestaltet es sich mit dem vielschichtigen Begriff der Kompetenz, der ebenfalls häufig verwendet wird. Es existieren verschiedene Definitionen und Auslegungen des Kompetenzbegriffs. In dieser Arbeit wird bewusst nicht die sprachliche und fachliche Kompetenz der Schüler*innen als Zielvariable ausgerufen, sondern die Veränderung von Teilen des Fachwissens und der Fachsprache untersucht.

\section{Die Rollen und die Bedeutung von Sprache für den Geographieunterricht}

Im Grundlagenteil wird über die verschiedenen Rollen von Sprache im Geographieunterricht referiert (vgl. Abschn. 2.2). Die Darstellung des dahingehend vorliegenden Forschungsstandes ist nicht gänzlich neu, zeigt aber in der Zusammenschau verschiedener Forschungsarbeiten die Bedeutsamkeit von Sprache im Geographieunterricht auf. Was dadurch theoretisch offensichtlich wird - Sprache ist wichtiger Bestandteil des Geographieunterrichts und steht in Zusammenhang mit dem Erwerb von Fachwissen - untersuchte die erörterte Studie empirisch für den Geographieunterricht. Weiter oben ist bereits beschrieben, dass es aus dem aktuellen Forschungsstand anderer Fächer sowie der Analyse der verschiedenen Rollen von Sprache im Geographieunterricht nahe liegt, sprachsensiblen Geographieunterricht durchzuführen. Die vorliegende Studie untermauert diese Forderung erstmals empirisch. Dass die schwächsten Leser*innen über alle Erhebungszeitpunkte hinweg deutliche Nachteile im Fachwissenstest haben, zeigt, wie wichtig Leseförderung auch im Fachunterricht ist. Ferner korrelieren die Lernzuwächse in 
Fachwissenstest und C-Test $(\mathrm{r}=.34, \alpha=.01)$ sowie die Lernzuwächse in Fachwissenstest und Profilanalyse miteinander $(r=.19, \alpha=.05)$. Dies stützt erneut den Befund, dass Sprache im Geographieunterricht hohe Bedeutung zukommt.

\section{Design-Kriterien für sprachsensiblen Geographieunterricht}

Auf Basis des Forschungsstandes wurden Design-Kriterien für sprachsensiblen Geographieunterricht abgeleitet. Dabei wurde interdisziplinär auf Möglichkeiten sprachsensiblen Fachunterrichts geblickt. Der Anspruch war, verschiedene Zugänge zu berücksichtigen und diejenigen, die sich bereits in anderen Fächern und Forschungsfeldern als fruchtbar erwiesen haben, auf den Geographieunterricht anzuwenden.

Das erste Kriterium „Guter Geographieunterricht“ ist gleichermaßen basal wie zur Diskussion gestellt - jedenfalls in seiner Operationalisierung. Weitgehend Konsens besteht darin, dass Geographieunterricht auf konstruktivistischem Lernverständnis basieren soll. Wellenreuther (2013) weist im Rahmen des Konstruktivismus auf die Notwendigkeit von direkter Instruktion hin. An dieser Stelle möchte ich diesen Aspekt explizieren. Konstruktivistischer Unterricht bedeutet nicht, die Schüler*innen mit dem zu Lernenden allein zu lassen, sondern sie durch Instruktion zu unterstützen (vgl. Abschn. 3.1).

Als weiteres zentrales Kriterium kristallisierte sich das sprachliche Scaffolding heraus. Dieses ist einerseits vor dem Hintergrund konstruktivistischen Lernverständnisses relevant und wird andererseits besonders in der Sprachdidaktik als notwendig für durchgängige Sprachbildung erachtet (vgl. Abschn. 3.3). In der Unterrichtsintervention wird bei der Operationalisierung des sprachlichen Scaffoldings nicht jeder sprachliche Bereich gleich stark beleuchtet. Vor allem bei der Textproduktion gibt die Unterrichtsreihe gestufte Hilfen im Sinne des Scaffoldings. Der mündliche Unterrichtsdiskurs (Mikro-Scaffolding) ist nicht explizit Untersuchungsgegenstand. Diese Schwerpunktsetzung ergibt sich aus dem Untersuchungsdesign und den entwickelten/adaptierten Erhebungsinstrumenten. Die Aufnahme von Gesprächen, was eine Untersuchung auf Mikro-Scaffolding-Ebene überhaupt erst ermöglicht hätte, war aus datenschutzrechtlichen Gründen nicht durchführbar, sodass Erhebungsverfahren genutzt wurden, die Schriftsprache fokussieren. Es ist erstrebenswert, in Anschlussforschung das sprachliche Scaffolding auf verschiedene Wege zu operationalisieren und auf seine Wirksamkeit hin zu prüfen (vgl. Abschn. 8.4).

Die Darstellungsvernetzung ist insbesondere für die Geographie ein wichtiges Design-Kriterium, da das Fach eine Vielzahl an verschiedenen (dis)kontinuierlichen Darstellungsformen aufweist. Auch bei diesem Kriterium ist eine vielseitige Operationalisierung möglich. 
Die Arbeit untersuchte den Einbezug der Erstsprache erstmalig in dieser Form für den Geographieunterricht und erwies sich in den Beobachtungen der Unterrichtsreihe sowie der Rückmeldung der Schüler*innen als sinnvoll, insbesondere hinsichtlich verschiedener motivationaler Aspekte. Genauere Untersuchungen hierzu müssten noch vorgenommen werden, da Motivation ein komplexes Konstrukt verschiedener Bestandteile und Einflussfaktoren ist.

Es ist abschließend herauszustellen, dass die Kriterien keinen Anspruch auf Vollständigkeit erheben. Die Bezugsdisziplinen sind gewichtet und von unterschiedlicher Relevanz; es sind durchaus andere gute Schwerpunktsetzungen und Kriterien denkbar und sinnvoll.

\section{Erhebungsinstrumente}

Ein zentraler Beitrag für weitere Forschung liegt in der Entwicklung und Adaption verschiedener Erhebungsinstrumente. Bisher noch nicht vorhanden war ein Fachwissenstest für das Fach Geographie zu den Themen Schalenbau der Erde und Plattentektonik. Ein selbst entwickelter sprachlich möglichst niedrigschwelliger Multiple-Choice-Test überprüft das Fachwissen. Der Test erwies sich in seiner Reliabilität und Validität als sehr zufriedenstellend und kann auch in der Folgeforschung eingesetzt werden.

Um die Fachsprache zu erheben, wurden zwei Messinstrumente adaptiert. Zum einen ist ein C-Test entstanden, der sehr ökonomisch in Erstellung, Auswertung und Interpretation ist und alle weiteren Gütekriterien gleichsam gut erfüllt. Den C-Test, der sonst vorwiegend in der Zweit- und Fremdsprachenforschung eingesetzt wird, für die Erhebung der Fachsprachlichkeit zu erheben, ist bisher nur in wenigen Studien erprobt und hat sich im Rahmen dieser Arbeit als ertragreicher Ansatz erwiesen. Da aber der C-Test insbesondere die lexikalische Seite von Fachsprache erfasst, wurde zusätzlich eine Profilanalyse eingesetzt und ausgewertet. Die Profilanalyse umfasst verschiedene Auswertungskategorien, die aus bisherigen Arbeiten übernommen oder angepasst wurden. Die Auswertung der Profilanalyse ist deutlich umfangreicher und komplexer als die des C-Tests, gleichzeitig ist es durch sie möglich, ein Bild der Fachsprachlichkeit der Schüler*innen zu erhalten, dass nicht nur die lexikalische Ebene von Sprache umfasst. Doch auch die Profilanalyse hat Schwächen. So berücksichtigt sie kaum die pragmatische Seite von Sprache und inhaltliche Korrektheit spielt eine nebengeordnete Rolle. Pragmatik und Inhalt finden jeweils in nur einer der 16 Auswertungskategorien Berücksichtigung. Gleichzeitig stellt die Isolation der inhaltlichen Gesichtspunkte den Versuch dar, die Konstrukte Fachsprache und Fachwissen möglichst unabhängig voneinander zu erheben. Eben zu dieser Diskussion um die Unterscheidung von Fachwissen und Fachsprache leistet die vorliegende Arbeit einen Beitrag. Durch die Erstellung 
und den Einsatz der Erhebungsinstrumente sowie die ausführliche Erörterung der verschiedenen sprachlichen Register entsteht mehr begriffliche Klarheit.

Neben den Erhebungsinstrumenten zu Fachsprache und Fachwissen wurden auch Items zur Selbsteinschätzung bezüglich des Fachwissens und der Fachsprache im Fach Geographie aus größeren Schulleistungsstudien auf den Geographieunterricht adaptiert (vgl. Abschn. 6.2). Alle selbst entwickelten bzw. adaptierten Erhebungsinstrumente wurden über zwei Design-Zyklen hinweg empirisch erprobt, zeigen hohe Erfüllung der Gütekriterien und sind daher auch für den Einsatz in etwaiger Folgeforschung geeignet.

\section{Empirische Evidenzen}

Ein zentraler Beitrag zur aktuellen Forschung liegt in der vorgelegten Dokumentation des empirischen Vorgehens, der Bereitstellung eines Datensatzes und der erkannten Evidenz (vgl. Abschn. 7.3-7.6). Die Dokumentation soll Lehrkräfte ermächtigen, selbstständig einen Zugang zu sprachsensiblem Geographieunterricht zu konstruieren und kann als Leitfaden für Unterrichtsentwicklung gelesen werden. Die zum Datensatz gehörigen Erhebungsverfahren können im Unterricht verwendet werden.

Der wesentliche Teil der Forschungsarbeit war es, die auf den Design-Kriterien beruhende sprachsensible Unterrichtsreihe hinsichtlich ihrer Wirksamkeit zu untersuchen. Zusammengefasst zeigen sich in Hinblick auf diverse Variablen und Bezugsgruppen Vorteile für diejenigen Schüler*innen, die sprachsensiblen Geographieunterricht erhalten haben. Die zentrale Erkenntnis ist, dass sprachsensibler Geographieunterricht sowohl den Fachwissenserwerb als auch die Fachsprachlichkeit der Schüler*innen besser fördert als nicht-sprachsensibler Geographieunterricht $(\mathrm{d}=.51, \alpha=.05, \beta-1=.80)$.

Bezüglich der bezugsgruppenspezifischen Untersuchungen zeigt sich, dass die schwächsten Leser*innen in der Experimentalgruppe hinsichtlich aller Variablen leichte, nicht signifikante Vorteile im Vergleich zur Kontrollgruppe haben. Es wird auch deutlich, dass die mittleren und guten Leser*innen noch stärkeren Zuwachs in Fachsprache und Fachwissen haben als die schwachen Leser*innen. Eine Erklärung hierfür ist der aus der Soziologie bekannte Matthäus-Effekt („,Denn wer da hat, dem wird gegeben, dass er die Fülle habe; wer aber nicht hat, dem wird auch das genommen, was er hat.“"Matthäus-Evangelium 25,29), wovon der erste Teil des Satzes auch in dieser Studie zum Tragen zu kommen scheint. Denn auch sprachstarke Schüler*innen zeigen in der Experimentalgruppe deutlich höhere Leistungszuwächse als in der Kontrollgruppe. Auch die sprachschwachen Schüler*innen profitieren von dem Treatment. Positiv formuliert zeigt sich in diesem Befund ein wesentlicher Vorteil von sprachsensiblem Geographieunterricht. Er dient nicht nur den 
(lese)schwächsten Schüler*innen, sondern ist hinsichtlich der betrachteten Merkmale wirksamer als nicht-sprachsensibler Geographieunterricht. Damit entkräften die dargestellten Ergebnisse Bedenken, dass bei einer sprachsensiblen Ausrichtung des Geographieunterrichts Fachlichkeit zu kurz kommt. Außerdem räumt er mit dem Missverständnis auf, dass sprachsensibler Geographieunterricht nur die Schwächsten fördert. Wenn binnendifferenzierter Unterricht möglichst gut gelingen soll, ist der Anspruch, dass alle gefordert werden. Dies bedeutet auch, dass sich die Unterschiede zwischen den Leistungsstarken und Leistungsschwachen nicht zwingend schmälern. Bezüglich der Unterschiede zwischen leseschwachen und -starken Schüler*innen zeigt sich in Bezug auf die Variable Fachsprache, dass der Schereneffekt in der Experimentalgruppe abgemildert werden konnte; für die Variable Fachwissen ist dieser Befund so nicht feststellbar. Im Gegenteil: Es zeigt sich, dass die Lesestarken der Experimentalgruppe höheren Leistungszuwachs haben als die Leseschwachen derselben Gruppe $(\mathrm{d}=-.63, \alpha=.05,1-\beta=.80)$. In der Kontrollgruppe fallen die Unterschiede im Fachwissenszuwachs zwischen Lesestärksten und Leseschwächsten nicht signifikant aus. Erst wenn Sprache als Hürde überwunden ist, können Lehrende und Lernende die Aufmerksamkeit auf das fachliche Lernen lenken. Die Befunde zeigen damit, dass Lesekompetenz nicht nur zentrale Voraussetzung für Schulerfolg im Allgemeinen ist, sondern ein Schlüssel für Teilhabe am Fachunterricht. Die Erkenntnisse werfen außerdem die Frage auf, ob nicht die schwächsten $30 \%$ der Leser*innen angepasste Lernziele für den Fachunterricht brauchen. Dass diese Überlegung angestellt werden kann, ist der genaueren Betrachtung der Stichprobe untergliedert nach Bezugsgruppen zu verdanken. An dieser Stelle offenbart sich - auch im Vergleich zu anderen empirischen Studien zu sprachsensiblem Fachunterricht - ein wesentlicher Vorteil der statistischen Untersuchungen in dieser Arbeit.

Weder zum ersten Erhebungszeitpunkt noch im Verlauf der weiteren Erhebungen lassen sich Unterschiede zwischen Schüler*innen mit Deutsch als Erst- oder Zweitsprache feststellen. Dies ist insofern ein erfreulicher Befund, als er darauf hindeutet, dass Kinder mit mehrsprachigem Hintergrund jedenfalls in dieser Kohorte keine signifikanten Nachteile haben und die entsprechenden Schulen bereits in irgendeiner Form auf die praktische Realität der multilingualen Schule reagieren.

Nach den Untersuchungen unklar bleibt die Rolle der Selbsteinschätzung. Diese Variable ist im Forschungsprozess als relevante Größe aufgefallen. Im ersten Design-Zyklus waren Korrelationen zwischen Geschlecht und Selbsteinschätzung zu erkennen. Die Mädchen schätzten im ersten Design-Zyklus ihre sprachlichen und fachlichen Kompetenzen schlechter ein als die Jungen, und das, obwohl sie in den entsprechenden Tests dazu leicht bessere Ergebnisse erzielten. Diese Diskrepanz war derart auffällig, dass die Selbsteinschätzung im zweiten Design-Zyklus 
im Prä-Post-Follow-up-Vergleich und nicht mehr nur zu t1 erhoben wurde. Für den zweiten Design-Zyklus ergeben sich allerdings keine signifikanten Korrelationen mehr und es bedarf weiterer empirischer Untersuchung. Dadurch, dass die Variable der Selbsteinschätzung erst im Forschungsprozess aufgetaucht ist, ist sie nicht explizit im Grundlagenteil aufgearbeitet. Dies sollte Gegenstand von Folgearbeiten sein. Die skizzierten Einsichten legitimieren und fordern weitere Forschung zur Wirksamkeit sprachsensiblen Geographieunterrichts in verschiedenen Formen der Operationalisierung.

\subsection{Konsequenzen und Empfehlungen für die Unterrichtspraxis}

Neben den oben dargestellten theoretischen und empirischen Erkenntnissen lassen sich aus der durchgeführten Studie diverse Konsequenzen für die unterrichtliche Praxis ableiten. Die Design-Kriterien für sprachsensiblen Geographieunterricht sind eine zentrale Praxisempfehlung. Die nachfolgenden Aspekte lösen allerdings den Blick davon und fassen weitere Aspekte der Arbeit für die praktische Umsetzung ins Auge.

\section{Leseförderung stärken}

Im Rahmen der Studie wurden die schwächsten $30 \%$ der Leser*innen eingehender betrachtet. Bei Analyse der differenziellen Treatmenteffekte in der sprachsensiblen Gruppe zeigt sich, dass die mittleren und guten Leser*innen stärker vom Treatment profitieren als die schwächsten Leser*innen. Dies könnte bedeuten, dass die Hilfen, die das sprachsensible Treatment anbietet, nicht auf angemessenem Niveau stattgefunden haben und noch niedrigschwelliger angesiedelt sein müssen. Darüber hinaus kann es sinnvoll sein, für die Leseschwächsten eigene Lernziele zu entwickeln. Dass die schwächsten Leser*innen nicht stärker profitieren als die mittleren und guten Leser*innen, kann auch daran liegen, dass im Rahmen der Studie nicht für jede Klasse individuelle Anpassungen der gestuften Hilfen vorgenommen werden konnten, um Vergleichbarkeit zwischen den Klassen zu garantieren. Sprachsensibler Geographieunterricht muss adaptiv wirken und kann dementsprechend nicht als abstrakte Methode pauschalisiert werden.

Insgesamt zeigt sich, dass die Anforderungen an Leseförderung in der Sekundarstufe I breiter diskutiert werden müssen. Für Lehrkräfte bedeutet das, dass Lesekompetenz in allen Fächern gefördert werden muss. Lesen anzuregen, auch 
das private Lesen, muss integraler Bestandteil jedes Unterrichts werden. Ein möglicher Zugang ist der Einbezug literarischer Texte im Fachunterricht. So gibt es zum Beispiel in der untersuchten sprachsensiblen Unterrichtsreihe einen Baustein, der Bezug auf Jules Vernes Reise zum Mittelpunkt der Erde nimmt. Die Lehrperson liest den Schüler*innen einen Auszug aus dem Buch vor; die Schüler*innen aktivieren ihre eigenen Vorstellungen zum Erdinneren, vergleichen sie mit der literarischen Aufbereitung und schließlich beschäftigen sie sich mit dem Erdinneren aus fachlicher Perspektive. Denkbar wäre auch, die Schüler*innen den Abschnitt selbst lesen zu lassen, allerdings stand in diesem Teil der Unterrichtsstunde die literarische Ästhetik im Fokus. Die Schüler*innen sollten sich auf den literarischen Text einlassen, ohne selbst lesen zu müssen, was für manche Schüler*innen möglicherweise eine zusätzliche Belastung darstellt.

\section{Schreiben als integraler Bestandteil des Geographieunterrichts}

Abgesehen von der notwendigen fächerübergreifenden Leseförderung plädiert diese Arbeit für mehr Schreiben im Geographieunterricht. Insbesondere unter Einbezug von Scaffolding und Darstellungsvernetzung zeigte sich das Schreiben in der Studie als Schlüssel zum fachlichen Verstehen. Dabei bezieht sich das Schreiben eben nicht primär auf Hefteinträge, sondern dient dem Erkenntnisgewinn selbst. Epistemisches Schreiben zeigt sich für sprachschwächere Schüler*innen als hilfreich in der Sprachförderung, da der Schreibprozess Zeit gibt, die in der gesprochenen Sprache nicht vorhanden ist. Außerdem nimmt es Druck, der durch Sprechen, insbesondere vor oder im Klassenverband durch die Anwesenheit (und Beurteilung) anderer vorhanden ist.

\section{Sprachliches Scaffolding}

Sowohl die angesprochene Lese- als auch die Schreibförderung sollten im Rahmen des sprachlichen Scaffoldings stattfinden. Die Untersuchungen der schwächsten $30 \%$ der Leser*innen zeigen, dass die in der Unterrichtsreihe dargebotenen Hilfen möglicherweise noch nicht ausreichend niedrigschwellig angesiedelt waren, um die schwächsten so gut zu fördern wie die stärksten Leser*innen. Durchgängige Sprachförderung wird nur dann möglich sein, wenn sowohl sprachschwache als auch sprachstarke Schüler*innen möglichst gleich stark gefördert werden können. Aufgrund der großen Heterogenität im Klassenzimmer ist dafür Binnendifferenzierung unabdingbar; dass diese nicht nur in Bezug auf fachliche Aspekte geschehen sollte, sondern unbedingt ebenfalls unter sprachlichen Gesichtspunkten, zeigt die Arbeit eindrücklich. In der Arbeit wenig beleuchtet wird sprachliches Scaffolding auf Ebene des mündlichen Unterrichtsdiskurses. Diese Ebene zu stärken, ist eine zentrale Unterrichtsempfehlung. 


\begin{abstract}
Abwendung vom monolingualen Habitus
Sprachliche Vielfalt ist das Fundament dieser Arbeit. Die Schüler*innen dieser Stichprobe $(\mathrm{N}=320)$ sprechen 35 verschiedene Sprachen. Obschon dies nicht repräsentativ für jede Schule im Bundesgebiet ist, so zeigt die hohe Sprachenvielfalt an, dass Mehrsprachigkeit längst zum schulischen Alltag gehört. Es ist Zeit, diese anzuerkennen und, mehr noch, als Ressource zu begreifen. Das heißt nicht, dass alle Lehrkräfte die Erstsprachen ihrer Schüler*innen sprechen oder verstehen können sollen. Bei dieser Empfehlung geht es viel eher darum, auch andere Erstsprachen als das Deutsche als Werkzeug zum fachlichen Verstehen zu begreifen und nicht ständig und überall auf der deutschen Sprache im Unterricht zu beharren.
\end{abstract}

\title{
Qualifizierungsangebot etablieren
}

All die dargelegten Empfehlungen für die unterrichtliche Praxis bilden nicht die aktuelle Ausbildungssituation oder gar den Unterrichtsalltag ab. Lehrkräfte werden momentan nicht ausreichend geschult oder schon im Lehramtsstudium mit sprachlichen Anforderungen im Geographieunterricht konfrontiert. Es bleibt eher dem Zufall überlassen, ob Studierende mit dem Thema Sprache im Klassenzimmer überhaupt in Kontakt kommen, insbesondere dann, wenn sie kein Sprachenfach studieren. Je nachdem unterscheidet sich der Qualifizierungsbedarf erheblich. Möglichkeiten sprachsensiblen Geographieunterrichts müssten integraler Bestandteil des Studiums sein. Alle Lehramtsstudierenden sollten darüber hinaus Module besuchen, die sich mit Sprache in der Schule allgemein sowie im Fachunterricht beschäftigt. Auch wenn es zunächst überrascht, können auch Sprachenfächer sprachsensibel oder nicht-sprachsensibel unterrichtet werden. So findet Grammatikunterricht in Deutsch meistens isoliert und nicht kontexteingebunden statt; Lektüre wird auch ohne gestufte Hilfen gelesen, Schreiben findet ebenfalls oft ohne Einbindung in Kontexte statt. Den Schüler*innen ist meist nicht klar, warum sie das, was sie im Deutschunterricht lernen, für andere Fächer benötigen. Das mag auch daran liegen, dass viele Textsorten, auf die im Deutschunterricht eingegangen wird, tatsächlich außerhalb dieses Rahmens keine Anwendung finden. Welche Hürden sprachlich bestehen, ist sprachlich kompetenten Sprecher*innen/Schreiber*innen/Leser*innen oft gar nicht bewusst und genau deshalb braucht es ein Qualifizierungsangebot, das für die sprachlichen Schwierigkeiten im Geographieunterricht sensibilisiert. Darüber hinaus bleibt die Rolle von guten Unterrichtsgesprächen oft unterbeleuchtet. Auch diese Arbeit bespricht das Mikro-Scaffolding zu wenig explizit und es braucht dringend Folgeforschung, die sich mit der Umsetzung von Mikro-Scaffolding im Geographieunterricht sowie angemessenen Schulungen für Lehrkräften beschäftigen. 
Qualifizierungsmaßnahmen für Lehrkräfte sowie die Implementierung von sprachsensiblem Geographieunterricht im Studium sind die wichtigsten Konsequenzen, die diese Arbeit aus ihren Ergebnissen ableitet. Ohne ein Angebot an Lehrkräfte für mehr Sprachsensibilität im Unterricht verbleiben die Erkenntnisse überwiegend auf wissenschaftlicher Ebene. Es sind daher Schulungen für Lehrkräfte sowie Seminare zu Sprachsensibilität an der Universität geplant. Gleichwohl ist anzumerken, dass es im Aus- und Fortbildungsbereich zu Sprachsensibilität, insbesondere auch zu Mehrsprachigkeit, positive Entwicklungen gegeben hat und sich sowohl im Studium als auch in Forschung und Lehrkräfteschulungen diverse Angebote etabliert haben (vgl. Peuschel/Burkard 2019b, S. 33-34).

\subsection{Grenzen}

Trotz der Beiträge, die die Arbeit zum aktuellen Forschungsstand und der praktischen Umsetzung sprachsensiblen Geographieunterrichts leisten kann, verbleiben einige Grenzen, die ich im Folgenden aufführen möchte. Auf einige Grenzen wird bereits bei Beurteilung der Gütekriterien kritisch hingewiesen (vgl. Abschn. 7.2).

\section{Einschränkungen der internen Validität}

Feldstudien sind anfällig für vielfältige Störfaktoren. Uhrzeit, Tag, persönliche Stimmung und andere Aspekte können in der Praxis nur schwer kontrolliert werden. Trotz diverser Maßnahmen für hohe interne Validität (vgl. Abschn. 7.2) konnten in dieser Studie nicht alle Störfaktoren isoliert werden. Eine zentrale Einschränkung der internen Validität sind die unterschiedlichen Eingangsvoraussetzungen von Kontroll- und Experimentalgruppe hinsichtlich der Variable Selbsteinschätzung. Diese Bedrohung der internen Validität wird dadurch abgemildert, dass bei der Beurteilung der Treatmenteffekte nur die Lernzuwächse beurteilt werden. Dennoch sind die unterschiedlichen Eingangsvoraussetzungen problematisch. Denn es ist möglich, dass der Lernzuwachs in der Experimentalgruppe durch höheres Vorwissen begünstigt wird und nicht allein auf das Treatment zurückzuführen ist. Darüber hinaus stört der Regressionseffekt die internale Validität. In der vorliegenden Studie werden nämlich Personen auf Individualebene hinsichtlich verschiedener Variablen untersucht; sie erfahren dann aber das Treatment auf Klassenebene. Dies hat zur Folge, dass nicht einwandfrei herausgestellt werden kann, ob Veränderungen in den Erhebungen zu t2 auf das Treatment zurückzuführen sind. 


\section{Einschränkungen der externen Validität}

Die größte Einschränkung der externen Validität liegt in der begrenzten Stichprobengröße. Aufgrund der begrenzten Anzahl an Proband*innen und der relativ großen Heterogenität innerhalb der Stichprobe sind nur eingeschränkte statistische Verfahren möglich. Die optimale Stichprobengröße wurde vor den Erhebungen für eine mittlere Effektgröße ermittelt (vgl. Abschn. 6.5). Ein entsprechend großes Sample konnte ebenfalls erstellt werden. Für Berechnungen auf Ebene der Gesamtstichprobe untergliedert nach Kontroll- und Experimentalgruppe sind die Gruppengrößen ausreichend. Dies liegt unter anderem darin begründet, dass die Treatmenteffekte mit mittlerer Größe deutlich ausfallen. Bei geringeren Effekten und zweiseitigen t-Tests wird die Hypothesenüberprüfung mit der vorhandenen Stichprobengröße problematisch. Dies gilt ebenfalls für die Betrachtung einzelner Bezugsgruppen. In den Untersuchungen wurde die Stichprobe hinsichtlich dreier Merkmale gesondert betrachtet (Geschlecht, Lesekompetenz, Erstsprache). Für manche Berechnungen zeigt sich an dieser Stelle bereits, dass die Stichprobengröße nicht ausreichend ist. Weitere Betrachtungen der Stichprobe untergliedert in andere Merkmale, wie zum Beispiel der Erstsprachen, wären Anknüpfungspunkte für Folgeforschung, können aber statistisch nicht mehr ausreichend plausibel abgebildet werden.

Einschränkend zur begrenzten Stichprobe sind eine teilweise hohe Anzahl an Fehlwerten in Design-Zyklus I zu nennen. Hierfür ist eine Reihe von Gründen ursächlich. Während der Fachwissenstest jeweils zu t1 und t2 in jeder Klasse durchgeführt wurde, Fehlwerte also allein auf das Fehlen einzelner Schüler*innen zu einem der Erhebungszeitpunkte zurückzuführen sind, wurden andere Erhebungen nur zu einem Erhebungszeitpunkt durchgeführt. Der Lesegeschwindigkeits- und verständnistest sowie der Fragebogen zur Erhebung der Kontrollvariablen wurden lediglich zu t1 vorgelegt, da davon ausgegangen wurde, dass sich diese zu t2 nicht verändert haben werden. Dies wurde ebenfalls für den zweiten Design-Zyklus so angenommen - mit dem Unterschied, dass die Skala zur Selbsteinschätzung im zweiten Design-Zyklus explizit im Treatment der Experimentalgruppe adressiert werden soll, da sich auffällige Korrelationen mit den abhängigen Variablen ergeben haben. Diese Skala wurde daher in Design-Zyklus II auch zu t2 und t 3 erhoben. Die Profilanalyse zur Messung der Fachsprache konnte in Design-Zyklus I aufgrund der mangelnden Schreibmotivation nicht in allen Klassen zu t2 erneut durchgeführt werden. Da das Design im Rahmen des explorativen Design-Based Research eine gewisse Flexibilität hinsichtlich kleiner Umstellungen während eines Zyklus zulässt, wurde die leistungsbezogene geographiespezifische Fachsprachlichkeit in der letzten zu erhebenden Klasse mittels eines C-Tests durchgeführt. Es liegen daher für Zyklus I nur Daten aus einer Klasse vor. Im zweiten Design-Zyklus konnte die Einschränkung hoher Fehlwerte behoben werden. 
Eine weitere Einschränkung der externen Validität liegt im Forschungsdesign begründet. Design-Based Research untersucht komplette Unterrichtssettings in einem Zusammenspiel vieler verschiedener Faktoren. Dies hat den Vorteil, möglichst praxisnah die Wirksamkeit ganzer Settings untersuchen zu können. Im Umkehrschluss bedeutet das aber auch, dass der Erfolg oder Misserfolg einer Intervention nicht auf einzelne, isolierte Variablen zurückzuführen ist, wie es in klassischer Grundlagenforschung im Rahmen von (quasi-)experimentellen Designs der Fall wäre.

\section{Einschränkungen der Konstruktvalidität}

Eine weitere Grenze des Forschungsprojekts stellen die nicht völlig trennscharf zu erhebenden Konstrukte Fachsprache und Fachwissen dar. So korreliert der C-Test mit dem Fachwissenstest. Dennoch ist eben in der Konstruktion der verschiedenen Erhebungsinstrumente im Vergleich zum vor dieser Arbeit bestehenden Forschungsstand ein Fortschritt zu sehen (vgl. Abschn. 8.1). Durch die eingehende Auseinandersetzung mit der Problematik der Trennschärfe im Grundlagenteil der Arbeit und der daraus abgeleiteten Konstruktion der Erhebungsinstrumente wird das Problem adressiert und abgemildert.

\section{Einschränkungen der Objektivität}

Einschränkungen in der Objektivität sind insbesondere für Design-Zyklus I der Studie aufzuführen. Die Treatments in Design-Zyklus I wurden von mir selbst durchgeführt. Dies ist einem hohen Grad an Objektivität abträglich, wurde aber aus forschungspraktischen Gründen als vertretbar eingeschätzt. Die zunächst geplante Videographie der Unterrichtsreihe und anschließende externe Bewertung der Objektivität konnte aus Datenschutzgründen nicht durchgeführt werden. Im Rahmen des explorativen Designs erschien es vertretbar, dass das Treatment im ersten Design-Zyklus von mir selbst durchgeführt wurde; so war es möglich, positive und negative Aspekte des Unterrichts aus erster Hand zu erfahren und im zweiten Design-Zyklus entsprechend zu überarbeiten. Es wurde darauf geachtet, dass das nicht-sprachsensible Treatment vor dem sprachsensiblen Treatment durchgeführt wurde, um zu verhindern, Elemente des sprachsensiblen Treatments in die Kontrollgruppe zu integrieren. Im zweiten Design-Zyklus wurden die Unterrichtsreihen von der eigentlichen Geographielehrperson durchgeführt. Vorab erhielten die Lehrpersonen eine 90-minütige Schulung zur entsprechenden Unterrichtsreihe. Mindestens die Hälfte der Unterrichtsreihe habe ich beobachtet, um sicherzustellen, dass keine Abweichungen vom zu unterrichtenden Treatment vorhanden waren. Darüber hinaus wurde ein Fragebogen für die Lehrkräfte eingesetzt, in dem diese 
Angaben zu ihren (sprachlichen) Lehrgewohnheiten machten. Dies erlaubte es, bedingt Rückschlüsse auf Unterschiede im Lehrverhalten ziehen zu können.

\section{Diagnose und Therapie}

Eine weitere mögliche Grenze dieser Studie liegt in der praktischen Implementierung der Forschungserkenntnisse. Bereits im Forschungsdesign selbst ist ein hoher Praxisanteil enthalten; mit der Schulung der Lehrkräfte sowie des zyklischen Vorgehens ist bereits bei Durchführung der Studie ein praktischer Nutzen vorhanden. Darüber hinaus ist es allerdings eine wichtige Aufgabe, die Forschungserkenntnisse aus dieser Arbeit nach ihrer Fertigstellung in die Praxis zu tragen. Andernfalls verbleiben die Ergebnisse eher in der Wissenschaft; eine bloße Diagnose ohne Therapie. Durch Implementierung sprachsensiblen Geographieunterrichts bereits im Studium sowie im Schulungsangebot für Lehrkräfte kann Sprachsensibilität bottom-up integraler Bestandteil des Schulalltags werden.

\subsection{Ausblick}

Bevor die Arbeit insgesamt abschließend zusammengefasst wird, geht der Blick nach vorne. Was folgt auf diese Studie? Welche Fragestellungen und Untersuchungsgegenstände ergeben sich aus der Arbeit? Wo ergeben sich weitere Potenziale für Replikationen oder Überarbeitungen?

\section{Qualifizierungsangebot}

Im vorangegangen Unterkapitel wird bei den Grenzen auf die Implementierung der Erkenntnisse in der Praxis eingegangen. Um dem zu begegnen, sollen ein Qualifizierungsangebot für Lehrkräfte sowie Seminare für Studierende anknüpfend an diese Arbeit erstellt und durchgeführt werden, um eine entsprechende Multiplikation der Erkenntnisse zu erreichen. Darüber hinaus sind Handreichungen und Praxisbeiträge in Zeitschriften denkbare Wege, um die Erkenntnisse einem Praxispublikum zugänglich zu machen. Außerdem ist in Zeiten des Digitalen an soziale Medien zu denken. In welcher Form welche Zielgruppe sinnvoll erreicht werden kann, ist zu diskutieren.

\section{Selbsteinschätzung als Hemmnis und Chance}

Im Laufe des Forschungsprozesses ist die Variable der Selbsteinschätzung als möglicherweise relevanter Faktor für den Aufbau von Fachwissen und Fachsprache aufgefallen. Dadurch, dass die Selbsteinschätzung nicht von Anfang an Zielgröße 
der Untersuchung war, sondern durch die Flexibilität von Design-Based Research im zweiten Design-Zyklus Berücksichtigung finden konnte, war die theoretische Einbettung und damit die Interpretation dieser Größe in geringerem Ausmaß möglich. In Anschlussfragestellungen ist anzuraten, diese Variable als Zielvariable sowohl theoretisch als auch empirisch umfassender zu beleuchten. So stellt sich die Frage, welche Rolle die Selbsteinschätzung beim Erwerb von Fachsprache und Fachwissen spielt und inwiefern sie im sprachsensiblen Treatment adressiert werden kann. Bedeutsam erscheinen auf Basis der Erkenntnisse dieser Arbeit Geschlechterunterschiede hinsichtlich der Selbsteinschätzung, vor allem in Verbindung mit der tatsächlichen Entwicklung der Ergebnisse im Fachwissenstest und in der Fachsprache. Auf theoretischer Ebene sind in diesem Kontext Forschungsarbeiten zur Über- und Unterschätzung der eigenen Fähigkeiten zu betrachten. Ferner sei darauf hingewiesen, dass Selbsteinschätzung und Motivation fächerübergreifende pädagogisch-psychologische Aspekte sind. Diese grundlegend anzuheben und damit dem fachlichen Leistungszuwachs Raum zu geben, erfordert interdisziplinäres Zuspiel und ist nicht allein aus der geographiedidaktischen Forschung heraus zu stemmen.

\section{Schüler*innenvorstellungen und Sprachsensibilität}

Die Arbeit hat deutlich herausgestellt, dass Fachsprache in hohem Maße metaphorisch geprägt ist. Metaphern sind allgegenwärtiger Teil von Sprache und können das Verständnis erleichtern; insbesondere aber im fachlichen Kontext können Metaphern auch zu fachlich unangemessenen Schüler*innenvorstellungen führen oder diese befördern. Vor diesem Hintergrund erscheinen Untersuchungen zur Bedeutsamkeit von Metaphern in der Fachsprache und Schüler*innenvorstellungen relevant, um sprachsensiblen Geographieunterricht fachlich und sprachlich angemessen zu gestalten.

\section{Fokus auf einzelne Kriterien von sprachsensiblem Geographieunterricht}

Eine Grenze von Design-Based Research ist, dass gesamte Settings untersucht werden, ohne am Ende des Forschungsprozesses den Erfolg oder Misserfolg auf einzelne Variablen attribuieren zu können (vgl. Abschn. 6.1). Die Arbeit konnte - unter Berücksichtigung all ihrer Grenzen - herausstellen, dass sprachsensibler Geographieunterricht wirksam hinsichtlich des Erwerbs von Fachwissen und Fachsprache ist. Warum das so ist, klärt diese Arbeit nicht, und insofern ist Anschlussforschung ratsam, die die einzelnen Design-Kriterien näher untersucht. Es stellt sich die Frage, ob und wie die einzelnen Faktoren isoliert voneinander in unterschiedlichen Operationalisierungsformen funktionieren. Denkbar ist, dass die einzelnen Kriterien nur im Zusammenspiel Wirksamkeit zeigen, oder aber, dass manche Kriterien weniger 
wirksam sind als andere. Auf Basis des Grundlagenteils sowie der Operationalisierung der Kriterien für die vorliegende Forschungsarbeit liegt die Vermutung nahe, dass insbesondere dem sprachlichen Scaffolding eine große Bedeutung beigemessen werden sollte. Mehrfach wurde im Verlauf der Arbeit dargestellt, dass das Mikro-Scaffolding nicht explizit in die Auswertung der Unterrichtsreihe einfließt, obwohl es in der aktuellen Forschung eine große Rolle spielt. Anschlussforschung sollte den Fokus daher auch auf Mikro-Scaffolding und damit den mündlichen Unterrichtsdiskurs richten.

\section{Beitrag zur Inklusion}

Inwiefern kann sprachsensibler Geographieunterricht einen Beitrag zur Inklusion leisten? Diese Frage wird in dieser Arbeit nicht beleuchtet, ist aber insbesondere im aktuellen bildungspolitischen Diskurs von hoher Relevanz. Sprachsensibler Geographieunterricht in der Form, wie er in der vorliegenden Studie betrachtet wird, sensibilisiert Lehrkräfte hinsichtlich der sprachlichen Herausforderungen, doch ist damit nicht auf Aspekte der Inklusion im eigentlichen Sinne eingegangen. In Folgeforschung könnte die Rolle von Sprache in der Debatte um angemessene Inklusionskonzepte Berücksichtigung finden.

\section{Bezugsgruppen genauer untersuchen}

Was die Arbeit bereits in einigen Berechnungen leistet, ist die Betrachtung von drei Bezugsgruppen, getrennt nach Geschlecht, Lesekompetenz sowie Deutsch als Erst- oder Zweitsprache. Eingehendere Untersuchungen dieser Bezugsgruppen sind für anschließende Fragestellungen von Interesse, um das sprachsensible Treatment weiter zu optimieren. So zeigte sich in dieser Studie, dass die sprachlichen Scaffolds für besonders schwache Leser*innen noch nicht niedrigschwellig genug gewesen sind und möglicherweise auch binnendifferenzierte Lernziele veranschlagt werden sollten. In diesem Kontext scheint es ebenfalls aufschlussreich, hinsichtlich des Gruppenmerkmals Lesekompetenz homogene Gruppen zusammenzustellen, um genauer definieren zu können, was die Eingangsvoraussetzungen sind und worin die Schwierigkeiten im Einzelnen bestehen. Bei der Bezugsgruppe Geschlecht zeigen sich je nach betrachteter Variable Risiken für Mädchen oder Jungen, anders als dies für die Lesekompetenz der Fall ist; das Risiko liegt deutlich auf Seiten der schwachen Leser*innen. Die Analyse nach Deutsch-als-Erst- oder Zweitsprachler*innen scheint in der Unterteilung noch nicht feingliedrig genug zu sein. Sinnvoll erscheint in diesem Zusammenhang eine genauere Analyse der Bezugsgruppe. Ein genaueres Bild der Eingangsvoraussetzungen könnte gezeichnet werden, wenn die Stichprobe untergliedert nach ihren Erstsprachen und nicht binär nach dem Merkmal 
Deutsch als Erstsprache strukturiert würde. Risikogruppen ließen sich so genauer aufschlüsseln und das Treatment könnte entsprechend optimiert werden.

\section{Sprachsensibilität in Bezug auf den gesellschaftspolitischen Kontext}

In dieser Arbeit wird Sprachsensibilität allein auf Fachsprache bezogen. Betrachtungsgegenstand sind also im Vergleich zur Alltagssprache differierende, erschwerende Sprachstrukturen. Sprachsensibilität wird aber, insbesondere im gesellschaftlich-politischen Diskurs oft auch im Kontext diskriminierungssensibler Sprache verwendet. Schon rein begrifflich sollte eine Auseinandersetzung mit dem Verständnis von Sprachsensibilität diskutiert werden. Inwiefern kann Sprachsensibilität beispielsweise nicht nur auf Aspekte der Fachsprache blicken, sondern auch einen sprachlich sensiblen Umgang mit Gender und Kultur schulen? Während des Forschungsprozesses im Rahmen von Design-Based Research, also bei der engen Zusammenarbeit mit den Lehrkräften, ist immer wieder evident geworden, dass Sprachsensibilität auch im gesellschaftspolitischen Kontext Relevanz hat. Es sei in diesem Zusammenhang noch mal deutlich gemacht, dass Sprachsensibilität ein außerordentliches Maß an Sprachreflexion erfordert und ermöglicht - sowohl auf Seiten der Lehrkräfte als auch auf Seiten der Schüler*innen. Ist der Reflexionsprozess über Fachsprache in Gang gesetzt, so ist es möglicherweise nur noch ein kleiner Weg dahin, Begrifflichkeiten, deren Verwendung und deren Bedeutungszuschreibung(en) zu reflektieren und sich für bewusste Sprachverwendung, die immer ein Abwägen zwischen Optionen bedeutet, zu entscheiden. Der Geographieunterricht ist für Sprachsensibilität prädestiniert - insbesondere zum Beispiel im Umgang mit Karten, Kolonialgeschichte und Zuschreibung(en) von Raum. Aspekte, die unter anderem in dem im Grundlagenteil erwähnten linguistic turn in das Augenmerk der Forschung gerückt sind.

\section{Gendergerechter Geographieunterricht}

Im Rahmen dieser Arbeit werden Genderunterschiede insbesondere in Hinblick auf die Selbsteinschätzung untersucht. Außerdem sind Genderunterschiede in Bezug auf unterschiedliche Interessen bezüglich verschiedener Themen im Geographieunterricht auszumachen, wie verschiedene Interessenstudien zeigen. Auch im Rahmen dieser Arbeit zeigte sich in den Beobachtungen, dass das Thema Schalenbau der Erde und Plattentektonik bei den Jungen auf größeres Interesse stieß als bei Mädchen. Das Schreiben von Texten sowie die Aufgaben im Rahmen der sprachlichen Scaffolds wurden aber mit mehr Begeisterung von den Mädchen durchgeführt. Die Kombination eben dieser beiden Bereiche, also ein physisch-geographisches Thema (Vorteile für die Jungen) sprachsensibel auszurichten (Vorteil für die Mädchen), 
könnte die klassischen Genderunterschiede, die die aktuelle Forschung feststellt, ausgleichen.

\section{Weitere Analysen}

In dieser Arbeit kamen insbesondere deskriptive und interferenzstatistische Verfahren zur Anwendung. In weiterer Folgeforschung lohnt darüber hinaus der Blick auf Mehrebenen- und Strukturgleichungsmodelle. In Mehrebenenmodellen könnten noch stärker auffällige Einzeldaten berücksichtigt werden. Strukturgleichungsmodelle fassen verschiedene Bedingungen der empirischen Untersuchung modellhaft zusammen und können die dargestellten Daten anders strukturiert zum theoretischen Ausgangspunkt weiterer Forschungsarbeiten machen; zum Beispiel könnte die Frage behandelt werden, welche Rolle Selbsteinschätzung für die Variablen Fachsprache und Fachwissen spielt.

Darüber hinaus sollten insbesondere für den Fachwissenstest als gänzlich neu entwickeltes Erhebungsinstrument noch strengere empirische Prüfungen der Eindimensionalität z. B. über Faktorenanalysen durchgeführt werden.

Vom Reifegrad der wissenschaftlichen Forschung fehlt im Kontext Plattentektonik und sprachsensibler Unterricht in Zukunft ein Längsschnittexperiment mit fortlaufendem Förderassessments (wöchentlich) und einer explizit designten Kontrollbedingung. Die Geographiedidaktik kann die verwendete designbasierte, zyklische Methode des Unterrichtsdesigns vermutlich auch in anderen Unterrichtskontexten und Klassenstufen relativ einfach einsetzen. So könnten Kommunikationsprozesse mit der Schulpraxis initiiert und vorläufige fachdidaktisch relevante Evidenzen für praktische Problemlösungen bewertet werden. 
Open Access Dieses Kapitel wird unter der Creative Commons Namensnennung 4.0 International Lizenz (http://creativecommons.org/licenses/by/4.0/deed.de) veröffentlicht, welche die Nutzung, Vervielfältigung, Bearbeitung, Verbreitung und Wiedergabe in jeglichem Medium und Format erlaubt, sofern Sie den/die ursprünglichen Autor(en) und die Quelle ordnungsgemäß nennen, einen Link zur Creative Commons Lizenz beifügen und angeben, ob Änderungen vorgenommen wurden.

Die in diesem Kapitel enthaltenen Bilder und sonstiges Drittmaterial unterliegen ebenfalls der genannten Creative Commons Lizenz, sofern sich aus der Abbildungslegende nichts anderes ergibt. Sofern das betreffende Material nicht unter der genannten Creative Commons Lizenz steht und die betreffende Handlung nicht nach gesetzlichen Vorschriften erlaubt ist, ist für die oben aufgeführten Weiterverwendungen des Materials die Einwilligung des jeweiligen Rechteinhabers einzuholen.

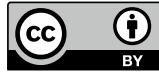

